\title{
CARACTERIZAÇÃO E CONTROLE DE QUALIDADE DE ÓLEOS DE COPAÍBA (Copaifera sp.) UTILIZANDO DETECÇÃO DE MARCADORES POR FATOR DE RETENÇÃO RELATIVA EM HPTLC
}

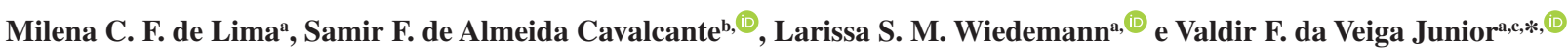 \\ aDepartamento de Química, Instituto de Ciências Exatas, Universidade Federal do Amazonas, 69080-900 Manaus - AM, Brasil \\ 'Instituto de Defesa Química, Biológica e Nuclear, Centro Tecnológico do Exército, 23020-470 Rio de Janeiro - RJ, Brasil \\ 'Departamento de Engenharia Química, Instituto Militar de Engenharia, 22290-270 Rio de Janeiro - RJ, Brasil
}

Recebido em 28/01/2020; aceito em 20/04/2020; publicado na web em 10/06/2020

\begin{abstract}
CHARACTERIZATION AND QUALITY CONTROL OF COPAIBA OILS (Copaifera sp.) BY RELATIVE RETENTION FACTOR BY HPTLC TO DETECTION OF BIOMARKERS. Copaiba oilresins present medicinal uses that demand the development of methodologies that could ensure the authenticity and guarantee the species differentiation. The usual way to differentiate the copaiba oils is analyze the terpene composition, mainly the diterpenic acids profile. Unlike caryophyllene and its oxide, copaiba diterpenes are not commercially available and lab obtention is expensive and time consuming. At this paper, a new methodology is presented that allows the fast and reliable detection of copaiba biomarkers using HPTLC retention factor relative to an appropriate standard. Several commercially available substances were analyzed and the monoterpene linalool oxide was chosen. The comparison of relative retention factors allowed the confirmation of caryophyllene and its oxides and the diterpenic acids copalic, hydroxy-copalic, acetoxycopalic, agathic, and hardwickiic. Calibration curve showed strong correlation $(|r| \geq 0.9962)$ with $R^{2} \geq 0.9928$.
\end{abstract}

Keywords: Copaifera multijuga; copaíba; quality control; HPTLC; Amazonia.

\section{INTRODUÇÃO}

Os óleos de copaíba possuem amplo uso nas indústrias de cosméticos e perfumes devido às suas diversas propriedades biológicas. A denominação adequada desses óleos como oleoresinas implica na abordagem apropriada para sua caracterização química, sendo de fundamental importância para o desenvolvimento de metodologias de controle de qualidade confiáveis, eficientes e de fácil execução. ${ }^{1}$ As oleoresinas de copaíba são obtidas diretamente das árvores do gênero Copaifera (Fabaceae), sem extração nem solvente. $\mathrm{O}$ óleo está presente em diversas estruturas da planta, mesmo nas plântulas, até nas raízes. Flui por canais lizógenos e esquizógenos, escoa e se acumula em bolsões naturais no tronco, formados por alargamentos destes canais. Quando os bolsões ficam muito cheios de óleo, como nas árvores mais antigas, provocam expansões (barrigas) que podem rachar o tronco, por onde a copaíba exsuda naturalmente. Não é raro encontrar animais se esfregando no tronco dessas árvores para curar suas feridas. Para obter quantidades maiores que podem alcançar vários litros, faz-se um furo no tronco da árvore com um trado, uma furadeira manual, até alcançar os bolsões. ${ }^{1}$

A composição dos óleos de copaíba, como de outras oleoresinas naturais, é de uma mistura de terpenos de variados pesos moleculares. Os mais leves, líquidos à temperatura ambiente, solubilizam os mais pesados, resinosos. No caso da oleoresina de copaíba, os terpenos leves são sesquiterpenos, principalmente o cariofileno e seus derivados e isômeros, e os pesados são ácidos diterpênicos de esqueletos labdano, clerodano e caurano. ${ }^{1}$ São também exemplos de oleoresinas naturais o incenso, a mirra e o breu (Burseraceae), nas quais os terpenos leves são majoritariamente os monoterpenos ${ }^{2,3} \mathrm{e}$ os pesados são triterpenos. ${ }^{4,5}$ Já as oleoresinas de pinheiros e coníferas são constituídas por monoterpenos (pinenos), cujo produto comercial é a terebintina, ${ }^{6}$ e os pesados são ácidos diterpênicos aromáticos, como os ácidos abiético e pimárico, formando uma mistura comercialmente conhecida como colofone. ${ }^{?}$

\footnotetext{
*e-mail: valdir.veiga@gmail.com
}

Essa composição das oleoresinas é muito pouco comum, comparada às dos demais óleos naturais. As oleoresinas de copaíba recebem diversas denominações (populares e também comerciais) de misturas naturais, como seiva (nutrientes hidrossolúveis), bálsamo (soluções naturais aquosas ricas em derivados de ácidos benzoico e cinâmico) e látex (emulsões de polímeros naturais com metabólitos especiais hidrossolúveis), mas o mais comum é que sejam descritas como óleos fixos, que são aqueles obtidos por prensagem das sementes e constituídos majoritariamente por triglicerídeos e ácidos graxos livres (com pequenas quantidades de carotenos, tocoferóis, fosfolipídios e fito-esteróis). ${ }^{89}$ Para os óleos fixos, as metodologias de controle de qualidade empregam índices relacionados aos ácidos graxos (lineares, não terpênicos), suas insaturações e oxidações. Quando os óleos de copaíba são analisados como se fossem óleos fixos, os sesquiterpenos são ignorados e os ácidos diterpênicos acabam sendo titulados-como se fossem ácidos graxos. ${ }^{10}$ No outro extremo, quando a oleoresina de copaíba é confundida com um óleo essencial (a mistura de substâncias voláteis obtida unicamente por arraste por vapor d'água), analisa-se somente os constituintes leves e voláteis, os sesquiterpeno; sendo ignorada a porção resinosa da copaíba, podendo representar a perda de informação sobre $70-80 \%$ de sua composição. ${ }^{11}$

Há diversas iniciativas para o controle de qualidade dos óleos de copaíba, mas poucas se baseiam em sua composição química completa. ${ }^{12}$ Entre sesquiterpenos e diterpenos, $\beta$-cariofileno e o ácido copálico correspondem aos marcadores químicos dos óleos de copaíba, uma vez que participam da composição química de todos os óleos já descritos. ${ }^{13} \mathrm{~A}$ análise somente dos sesquiterpenos é a mais comum, com duas abordagens usuais: relatar o total de substâncias voláteis (oxidadas ou não); ${ }^{11}$ ou a quantificação do cariofileno e o seu óxido. ${ }^{14}$ Já nas análises somente dos diterpenos, as estratégias enfrentam diversos desafios que vão da solubilidade à necessidade de derivatização (esterificação) na cromatografia a gás (GC), a dificuldade de eluição em tempos curtos em fase reversa em cromatografia líquida de alta eficiência (HPLC) e, principalmente, de obtenção de padrões. ${ }^{15}$ Enquanto os sesquiterpenos variam grandemente em quantidade, os ácidos diterpênicos variam também qualitativamente 
entre espécies. Em C. reticulata, por exemplo, os óleos de copaíba são usualmente constituídos em média por $50 \%$ de sesquiterpenos e $50 \%$ de ácidos diterpênicos. ${ }^{16} \mathrm{Já}$ em C. multijuga, apenas 3\% a 9\% da composição química dos óleos é constituída por ácidos diterpênicos. ${ }^{17}$ Entre as técnicas analíticas empregadas recentemente, destacam-se a CLAE-EM e o RMN. ${ }^{15}$

Uma das grandes evoluções das técnicas cromatográficas, especialmente da cromatografia a gás, foi o aumento da resolução que permitiu a análise de misturas complexas. Com a necessidade de identificação sem isolamento, trabalhos pioneiros como de Kóvats e van Der Dool-Kratz empregaram substâncias de referência facilmente obtidas, como séries homólogas de hidrocarbonetos lineares, para comparação dos tempos de retenção. ${ }^{18}$ Em outros estudos, um único padrão, presente ou não na amostra, foi empregado como referência de tempo de retenção, como na identificação de esteróis de esponjas de água-doce empregando colesterol como referência e índices de retenção relativos a ele. ${ }^{19} \mathrm{Na}$ cromatografia em camada delgada (TLC) é o fator de retenção (Rf) o parâmetro sensível a diversas condições experimentais, tais como: concentração do analito, variações na eluição e a composição das fases móvel e estacionária. $\mathrm{O}$ fator de retenção relativo a esse padrão $\left(\mathrm{Rf}_{\mathrm{R}}\right)$ permite estabelecer uma relação entre o analito e o padrão, minimizando interferências. O emprego de substâncias de referência em TLC é pouco explorada, mas tem sido mais comum na cromatografia em camada fina de alto desempenho (HPTLC), por sua maior eficiência e resolução. ${ }^{20}$

As dificuldades de análise da mistura complexa da oleoresina de copaíba, especialmente pelas necessidades de derivatização e ausência de padrões de referência dos diterpenos, motiva a elaboração de novas metodologias, principalmente empregando padrões de fácil acesso. No presente estudo foi desenvolvida uma metodologia para a detecção de seus principais biomarcadores empregando HPTLC para obtenção do $\mathrm{Rf}_{\mathrm{R}}$ destes terpenos tendo o óxido de linalol como referência.

\section{MATERIAIS E MÉTODOS}

\section{Reagentes}

Os ácidos diterpênicos empregados são padrões secundários, foram isolados de oleoresinas de copaíba (Copaifera-sp) em trabalhos anteriores do grupo de pesquisas e caracterizados por técnicas de Ressonância Magnética Nuclear $\left({ }^{1} \mathrm{H} \mathrm{e}{ }^{13} \mathrm{C}\right)$. Os ácidos agático, copálico, hidróxi-copálico e acetóxi-copálico foram obtidos da oleoresina de $C$. multijuga, enquanto os ácidos hardwickiico e poliáltico foram isolados de C. reticulata. ${ }^{21}$ Os padrões de $\beta$-cariofileno ( $80 \%$ ), óxido de cariofileno $(95 \%)$ e óxido de linalol $(\geq 97 \%)$ foram adquiridos comercialmente da Sigma Aldrich. Como fase móvel no sistema de HPTLC utilizou-se hexano e acetato de acetila, e para solubilização dos padrões utilizou-se uma mistura de diclorometano e acetato de etila (1:1), todos de grau P. A. (TEDIA).

\section{Análise em HPTLC}

Os padrões terpênicos foram analisados por HPTLC individualmente e em mistura. As análises individuais foram realizadas em diferentes concentrações de cada biomarcador $\left(1 \mu \mathrm{g} \mathrm{mL} \mathrm{m}^{-1}\right.$ a $\left.1000 \mu \mathrm{g} \mathrm{mL} \mathrm{m}^{-1}\right)$ e as concentrações que mostraram melhor resolução e detecção nas análises foram selecionadas para compor a mistura de padrões terpênicos. A mistura de padrões foi constituída pelos ácidos copálico (250 $\left.\mu \mathrm{g} \mathrm{mL}^{-1}\right)$, hidróxi-copálico $\left(100 \mu \mathrm{g} \mathrm{mL}^{-1}\right)$, acetóxi-copálico (100 $\left.\mu \mathrm{g} \mathrm{mL}^{-1}\right)$, agático $\left(100 \mu \mathrm{g} \mathrm{mL}^{-1}\right)$, hardwíckiico $\left(100 \mu \mathrm{g} \mathrm{mL}^{-1}\right)$, poliáltico $\left(250 \mu \mathrm{g} \mathrm{mL}^{-1}\right)$ e pelo óxido de cariofileno $\left(100 \mu \mathrm{g} \mathrm{mL}^{-1}\right)$. As soluções padrão dos biomarcadores foram preparadas em acetato de etila (P.A.) a partir da solução estoque de cada biomarcador terpênico
(1 mg mL $\mathrm{m}^{-1}$ ). A separação cromatográfica foi realizada utilizando um sistema de HPTLC equipado com aplicador de amostras semiautomático, software integrado Win-CATS versão 1.4 .3 e visualizador de placas. As análises foram realizadas em placas de HPTLC, de alumínio, pré-revestidas com gel de sílica 60F254 (10,0 x 5,0 cm, Merck, Alemanha). As placas foram pré-lavadas com metanol, secas e ativadas em estufa de ar quente a $100{ }^{\circ} \mathrm{C}$ por $5 \mathrm{~min}$. As alíquotas de $10 \mu \mathrm{L}$ dos padrões (nas concentrações descritas anteriormente) foram carregadas como bandas de $6 \mathrm{~mm}$ nas placas de HPTLC, com dispositivo de aplicação semiautomático (CAMAG Linomat 3) com uma seringa de $500 \mu \mathrm{L}$ (Hamilton, Bonaduz, Suíça). As posições de aplicação foram definidas a $11 \mathrm{~mm}$ dos lados (esquerdo e direito) e $5 \mathrm{~mm}$ das extremidades inferior e superior da placa. A fase móvel foi preparada no momento da eluíção, com 10 minutos de saturação da cuba e constituída por uma mistura binária de hexano:acetato de etila (7:3). Após a aplicação dos padrões, as placas foram reveladas na luz ultravioleta ( $254 \mathrm{~nm}$ e $366 \mathrm{~nm}$ ), após a borrifação com vanilina sulfúrica ou com etanol sulfúrico, seguida de visualização no ultravioleta $(366 \mathrm{~nm})$. Posteriormente, foram secas usando ar quente e fotodocumentadas no TLC-VISUALIZER 2 (CAMAG). Os Rf's das bandas cromatográficas foram obtidos utilizando o software Win-CATS versão 1.4.3, integrado ao sistema de HPTLC (CAMAG). Definiu-se os pontos inicial e final da corrida cromatográfica e o ponto central da banda cromatográfica de interesse, gerando o valor do Rf com precisão de duas casas decimais.

\section{Fator de retenção relativo $\left(\mathbf{R f}_{\mathrm{R}}\right)$}

O Rf relativo $\left(\mathrm{Rf}_{\mathrm{R}}\right)$ foi obtido pelo desenvolvimento da curva analítica do óxido de linalol em 6 concentrações distintas $(40,70$, $100,250,750$ e $1000 \mu \mathrm{g} \mathrm{mL}^{-1}$ ) e aplicados na mesma placa com uma mistura dos padrões terpênicos $\left(100 \mu \mathrm{g} \mathrm{mL} \mathrm{m}^{-1}\right)$. Foram realizadas 10 réplicas da curva analítica em quatro dias distintos sob as mesmas condições instrumentais e cromatográficas. As placas foram eluídas em um sistema de HPTLC equipado com aplicador de amostras semiautomático, conforme descrito anteriormente. $\mathrm{O}$ experimento foi conduzido sob as seguintes condições: dimensões da placa: $11 \mathrm{~cm}$ x $6 \mathrm{~cm}$ (5 cm de eluição cromatográfica); comprimento da banda: $6 \mathrm{~mm}$; base inferior: $5 \mathrm{~mm}(0,5 \mathrm{~cm})$; base superior: $5 \mathrm{~mm}$ $(0,5 \mathrm{~cm})$; tempo de saturação da cuba: 20 minutos; tempo de ativação da placa na estufa: 5 minutos, a $100{ }^{\circ} \mathrm{C}$. Os padrões foram aplicados em cromatoplaca de HPTLC $(10 \mu \mathrm{L})$, eluídos em hexano:acetato de etila (7:3) e revelados em etanol sulfúrico, sob aquecimento por 7 minutos. Os fatores de retenção relativos foram obtidos pela razão entre o Rf dos padrões terpênicos e o Rf do óxido de linalol nas diferentes concentrações.

\section{Análise estatística}

O tratamento estatístico dos dados foi realizado usando o software R. ${ }^{22}$ Para construção e análise do ajuste das curvas analíticas utilizouse as funções lm (Linear Model), bptest (teste de Breusch Pagam para homogeneidade dos resíduos) e qqPlot (teste para normalidade dos resíduos).

\section{RESULTADOS E DISCUSSÃO}

A busca pela caracterização e controle de qualidade dos óleos de copaíba empregando técnicas mais simples, rápidas e baratas que aquelas publicadas recentemente visou eliminar os maiores impeditivos da maioria das técnicas: analisar a oleoresina sem precisar de derivatização e mesmo na ausência dos biomarcadores para comparação, em especial dos diterpenos. De posse desses padrões, 
resultado das investigações e isolamentos realizados com os óleos de copaíba pelo grupo de pesquisas, procedeu-se o estudo de diversos sistemas por HPTLC. Para otimizar as análises dos biomarcadores da copaíba, foram pesquisadas diferentes condições de saturação e ativação das placas cromatográficas, de inserção das amostras e de composição de fase móvel. Encontradas as condições ótimas, os Rf dos biomarcadores tendo sido obtidos, buscou-se entre dezenas de substâncias mais facilmente acessíveis comercialmente, o padrão de comparação mais eficiente, que eluisse em região diferente dos biomarcadores, evitando sobreposições (resultados não apresentados). Com o padrão definido para relacionar seu $\mathrm{Rf}$ aos dos biomarcadores terpênicos, procedeu-se os estudos analíticos de repetição e variação das concentrações.

\section{Caracterização química dos biomarcadores da oleoresina de copaíba por HPTLC}

Os ácidos copálico $\left(250 \mu \mathrm{g} \mathrm{mL}^{-1}\right)$, hidróxi-copálico, $\left(100 \mu \mathrm{g} \mathrm{mL}^{-1}\right)$, acetóxi-copálico $\left(100 \mu \mathrm{g} \mathrm{mL} \mathrm{m}^{-1}\right)$, agático $\left(100 \mu \mathrm{g} \mathrm{mL}^{-1}\right)$, hardwickiico $\left(100 \mu \mathrm{g} \mathrm{mL}^{-1}\right)$, poliáltico $\left(250 \mu \mathrm{g} \mathrm{mL}^{-1}\right)$ e o óxido de cariofileno (100 $\left.\mu \mathrm{g} \mathrm{mL}^{-1}\right)$ foram analisados individualmente por HPTLC nas diferentes concentrações, volume de aplicação, sistemas de eluição e revelação para a caracterização de seus fatores de retenção (Rf), otimizando as condições de eluição que permitissem sua separação com resolução superior a 2,0. Diversos sistemas eluentes foram testados, solventes simples, misturas binárias e ternárias, assim como vários sistemas de visualização. Obtidas as condições ótimas, todos os padrões foram reunidos, formando o que foi chamado de mistura de padrões. Somente os ácidos hardwickiico $(\mathrm{Rf}=0,40)$ e poliáltico $(\mathrm{Rf}=0,39)$ apresentaram Rf's muito próximos, inviabilizando a caracterização adequada por HPTLC desses biomarcadores reunidos. A composição da fase móvel que apresentou a melhor separação dos biomarcadores foi constituída por hexano:acetato de etila 7:3, com volume de aplicação de $10 \mu \mathrm{L}$ de cada padrão. A melhor visualização das manchas foi alcançada com a revelação em etanol sulfúrico a $10 \%$, seguido de aquecimento por 5 minutos e visualização na luz ultravioleta com comprimento de onda $366 \mathrm{~nm}$ (Figura 1).

Apesar da proximidade entre os Rf's dos ácidos poliáltico e hardwickiico, eles foram mantidos na mistura de padrões terpênicos, pois são substâncias relevantes que podem auxiliar na discriminação das oleoresinas do gênero Copaifera. Cada oleoresina apresenta características específicas de sua espécie e, portanto, apenas em alguns casos, como em $C$. duckei Dwyer e C. guianensis Desf. são relatadas a presença mútua dos ácidos poliáltico e hardwickiico em concentrações muito próximas. ${ }^{23}$ No entanto, o poliáltico é o ácido diterpênico presente em maior concentração nos óleos de $C$. officinalis (Jacq.) L. ${ }^{24}$ e nos óleos de C. lucens Dwyer, ${ }^{23}$ com $11,0 \%$ e 70,0\% de concentração relativa, respectivamente. Por outro lado, o hardwickiico é um dos principais ácidos diterpênicos detectados nas oleoresinas de $C$. langsdorffii Desf., C. cearensis Huber ex Ducke e C. reticulata Ducke. ${ }^{23}$ A presença dos dois biomarcadores na mistura de padrões terpênicos mostra claramente suas similaridades químicas e os cuidados a serem tomados na caracterização dos ácidos poliáltico e do hardwíckiico, evitando conclusões precipitadas. Há relatos que indicam o poliáltico como constituinte ácido majoritário em C. officinalis (Jacq.) L. $(11,69 \%)^{24} \mathrm{e}$ curiosamente também há relatos para a mesma espécie sugerindo o hardwickiico como o ácido presente em maior concentração. ${ }^{23}$

As análises iniciais dos biomarcadores incluíram o $\beta$-cariofileno e o ácido caurenóico. Entretanto, dificuldades na coeluição com o ácido copálico nas análises em HPTLC, dificultaram a utilização do ácido caurenóico nesse experimento. A baixa absorção do $\beta$-cariofileno no ultravioleta e a limitação de massa dos ácidos copálico e hardwickiico, inviabilizaram a utilização do $\beta$-cariofileno na mistura de padrões para a caracterização química dos biomarcadores por HPTLC (Figura 1). Apesar do $\beta$-cariofileno não constar na mistura de padrões, o desenvolvimento da curva analítica para esse biomarcador foi realizado com êxito. Por se tratar do principal biomarcador terpênico detectado nos óleos de copaíba, sua caracterização através do $\mathrm{Rf}_{\mathrm{R}}$ é de grande relevância e aplicação. A mistura de padrões possibilitou identificações preliminares rápidas, sem a necessidade do isolamento. Apesar da boa resolução observada no cromatograma obtido por HPTLC, as similaridades estruturais de alguns biomarcadores podem resultar em coeluições, como ocorreu com os ácidos caurenóico e copálico (não apresentado) e hardwickiico e poliáltico (Figura 1). No entanto, obviamente esta mistura de padrões não está disponível aos pesquisadores/empresas que realizam os ensaios de controle de qualidade dos óleos de copaíba para certificação de qualidade e detecção de adulterações, sendo necessária a adição de um padrão para comparação.

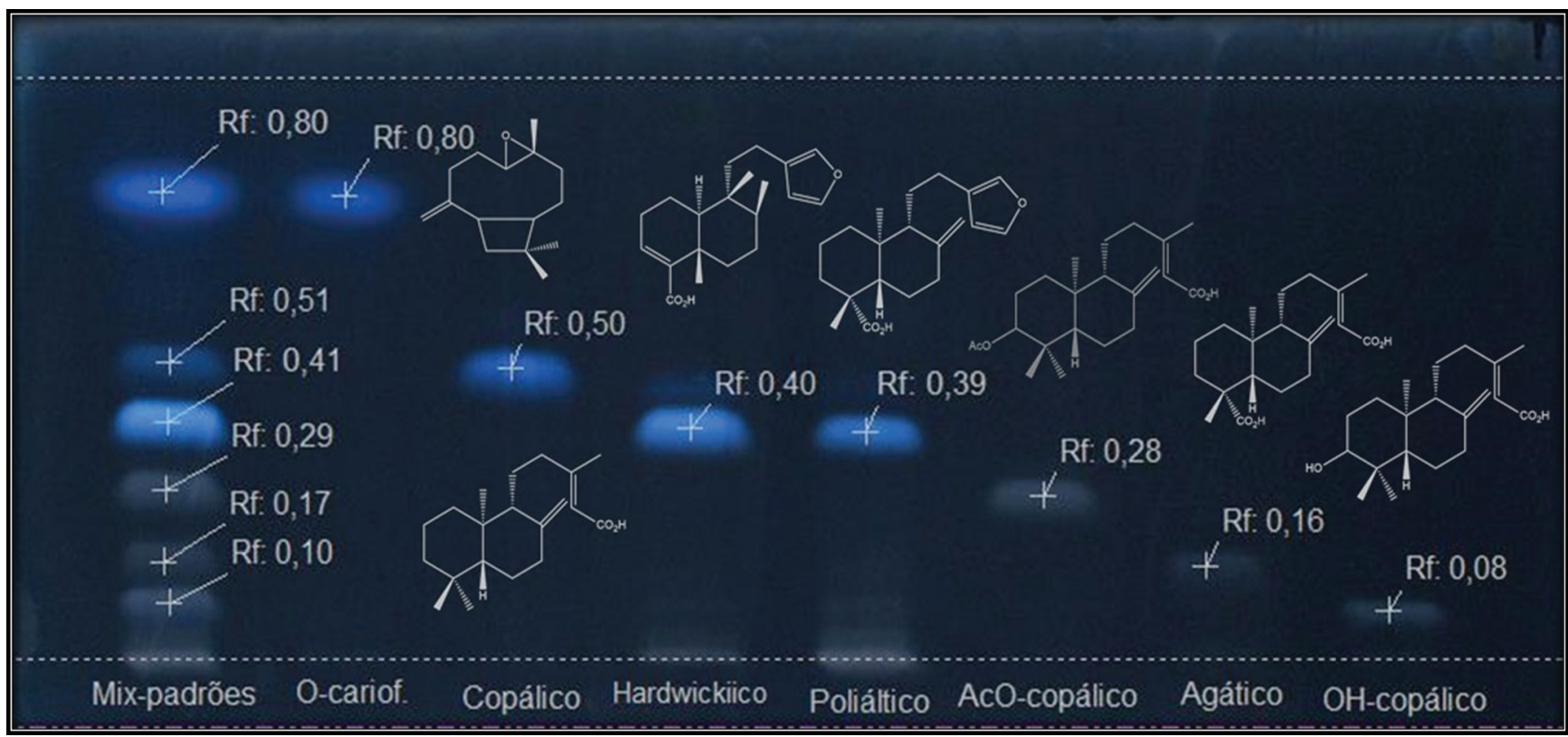

Figura 1. Perfil cromatográfico dos principais biomarcadores terpênicos detectados em óleos de copaíba (Copaifera sp) por HPTLC 
Fator de retenção relativo $-\mathbf{R f}_{\mathrm{R}}$

Entre diversos padrões de flavonoides, esteroides, derivados de ácido benzoico, ácidos graxos e terpenos que foram analisados com relação ao seu fator de retenção em condições próximas àquela que permitiu a observação dos principais biomarcadores dos óleos de copaíba, o óxido de linalol foi a substância que se mostrou mais adequada em termos de polaridade, estabilidade e repetibilidade das análises.

Vários padrões foram experimentados para o desenvolvimento do fator de retenção relativo $\left(\mathrm{Rf}_{\mathrm{R}}\right)$. Entre os padrões utilizados, muitos foram descartados por sua baixa polaridade e outros por coeluírem com os biomarcadores terpênicos presentes nos óleos de copaíba. O benzoato de benzila e o caempferol são alguns dos padrões testados para o desenvolvimento das curvas analíticas. No entanto, apesar do benzoato de benzila interagir menos com a fase estacionária e apresentar melhor banda em relação ao óxido de linalol, ele coeluiu com o óxido de cariofileno, inviabilizando sua utilização, além de apresentar uma baixa absorção na detecção por radiação na região do ultravioleta. A alta polaridade do caempferol contribuiu para fortes interações com a fase estacionária, inviabilizando também sua utilização. Apesar do óxido de linalol apresentar pequenas interações com a fase estacionária, ele não apresentou coeluição com nenhum dos biomarcadores presentes nos óleos de copaíba.

Estabelecendo o fator de retenção do óxido de linalol, o fator de retenção relativa dos biomarcadores de copaíba é obtido dividindo seus valores pelo valor do fator de retenção do óxido de linalol (Figura 2). Assim, as substâncias que eluem mais no sistema cromatográfico otimizado, mais apolares, e que eluíram mais que o óxido de linalol, possuem fator de retenção relativo a ele maiores que 1 . Isso ocorreu para o $\beta$-cariofileno e seu óxido. $O$ fator de retenção relativo do próprio óxido de linalol tem que ser 1,00. Já os ácidos carboxílicos diterpênicos, mais polares que o padrão e que ficaram mais retidos, eluindo menos, todos com valores de $\mathrm{Rf}$ menor, tiveram $\mathrm{Rf}_{\mathrm{r}}$ menores que 1 .

O experimento foi conduzido com o desenvolvimento da curva analítica dos biomarcadores em relação ao óxido de linalol, conforme detalhado na Figura 1S (Material Suplementar). A seleção das concentrações do óxido de linalol para o desenvolvimento da curva analítica foi baseada em estudos preliminares, que incluem o desenvolvimento de 9 placas feitas no HPTLC em 7 concentrações, para otimização das condições de análise. As curvas analíticas foram obtidas pelo desenvolvimento de 10 placas cromatográficas obtidas no HPTLC, sob as mesmas condições instrumentais $(40,70,100$, 250, 750,1000 $\mu \mathrm{g} \mathrm{mL}^{-1}$ ), gerando um total de 60 medições do $\mathrm{Rf}_{\mathrm{R}}$ para cada biomarcador terpênico. As curvas analíticas foram obtidas através de uma regressão linear em função do logaritmo neperiano (Ln) do óxido de linalol, cuja expressão matemática é descrita por

$$
\mathrm{Y}(\mathbf{x})=\mathrm{a}+\mathrm{b}^{*} \operatorname{Ln}(\mathbf{x})
$$

em que $\mathrm{Y}(\mathrm{x})$ representa o $\mathrm{Rf}_{\mathrm{R}}$ do biomarcador e $\mathrm{x}$ a concentração do óxido de linalol. Para cada curva, o coeficiente de explicação, $\mathrm{R}^{2}$, obtido foi sempre maior que $99 \%$, indicando que a variação na concentração do óxido de linalol é suficiente para explicar a variação no $\operatorname{Rf}_{R}$ dos biomarcadores. Em valores absolutos, o coeficiente de correlação, r, foi maior que 0,996 , sugerindo uma excelente relação log-linear (Material Suplementar, Tabela 1S). A significância da regressão mostrou-se forte (valor $\mathrm{p}<0,01$ ) para todos os padrões terpênicos. A homocedasticidade dos resíduos e a ausência de outliers também foram verificados, respectivamente, através do teste de Breush-Pagan e da análise dos resíduos padronizados (Material Suplementar, Figuras $2 \mathrm{~S}$ e $3 \mathrm{~S}$ ), evidenciando um bom ajuste da equação (1).

A metodologia desenvolvida $\left(\operatorname{Rf}_{R}\right)$ possibilitou identificações rápidas e confiáveis $(\mathrm{CV}<2 \%)$ por meio da HPTLC, que pode ser facilmente adaptada à TLC, que é uma técnica simples, de baixo custo, fácil manuseio e acessível a maioria dos laboratórios de produtos naturais. A obtenção do $\mathrm{Rf}_{\mathrm{R}}$ corresponde a análises preliminares, que inicialmente dispensam a utilização de técnicas onerosas como a GC-MS e HPLC-MS. Sob as condições experimentais desenvolvidas neste trabalho (concentração do padrão de referência e sistema de eluição), é possível usar o óxido de linalol como padrão para realizar identificações rápidas em óleos de copaíba.

Como resultado das análises pode-se sugerir para a detecção dos biomarcadores dos óleos de copaíba que uma análise por HPTLC seja realizada com o óleo e com o padrão de referência, o óxido de linalol, no intervalo de concentrações avaliadas $\left(40 \mu \mathrm{g} \mathrm{mL}^{-1}-750 \mu \mathrm{g} \mathrm{mL}^{-1}\right)$. Após a obtenção do $\mathrm{Rf}_{\mathrm{R}}$ que consiste na simples razão do biomarcador em relação ao óxido de linalol, basta consultar a Tabela 1 e associar os valores de $\mathrm{Rf}_{\mathrm{R}}$. Essa tabela foi desenvolvida para auxiliar a rápida identificação dos biomarcadores de copaíba. Através dela, sugerimos a utilização dos limites inferiores e superiores $\operatorname{dos} \mathrm{Rf}_{\mathrm{R}} \mathrm{de}$

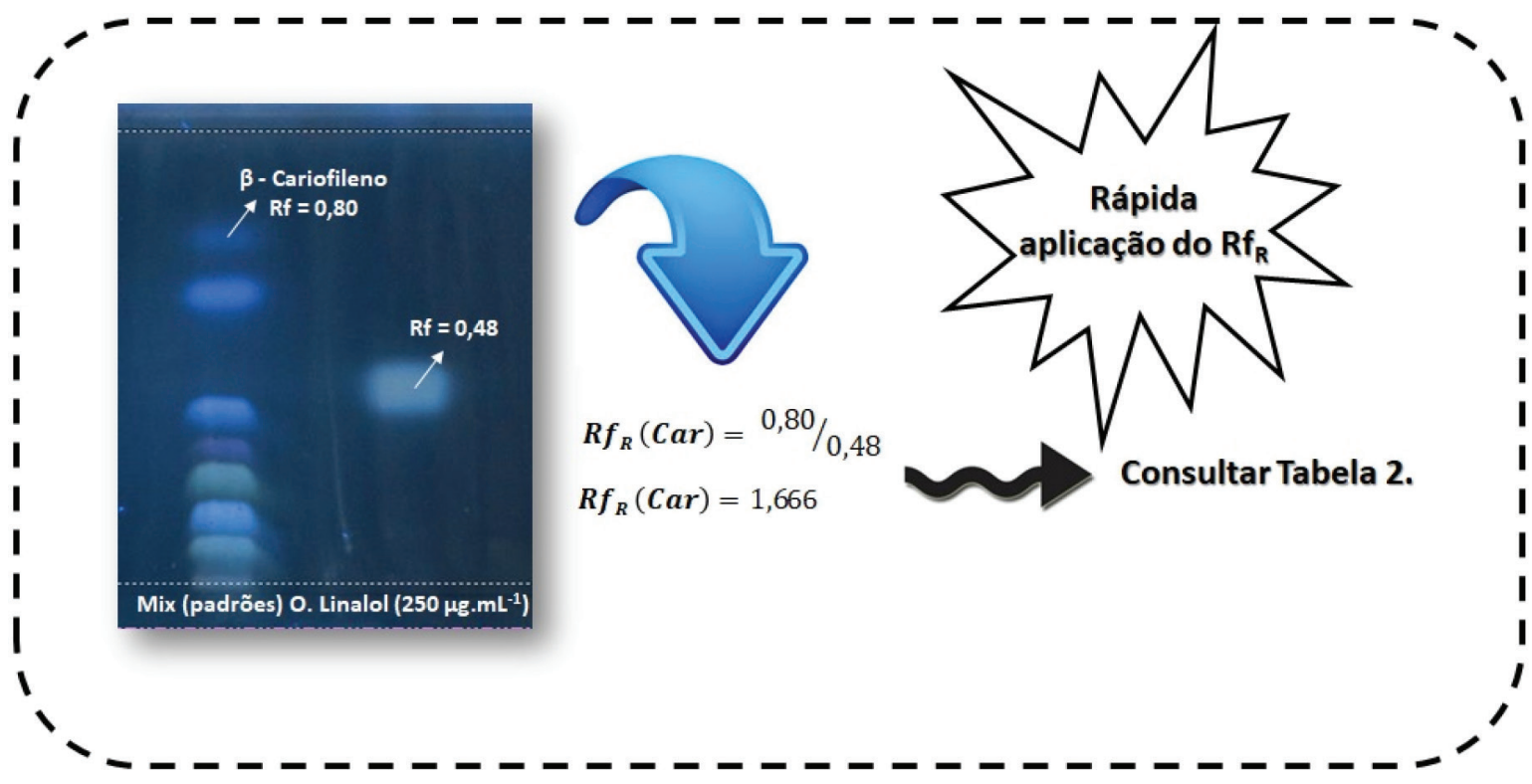

Figura 2. Ilustração prática da aplicação do fator de retenção relativo $\left(R f_{R}\right)$ 
Tabela 1. Limites de predição do $\mathrm{Rf}_{\mathrm{R}}$ para identificação de biomarcadores terpênicos em óleos de copaíba (Copaifera-sp) de acordo com a concentração do óxido de linalol

\begin{tabular}{cccccccc}
\hline $\begin{array}{c}\text { Concentração } \\
\left(\mu \mathrm{g} . \mathrm{mL} \mathrm{H}^{-1}\right.\end{array}$ & B-cariofileno & Óxido cariofileno & Ácido copálico & Ácido poliáltico & $\begin{array}{c}\text { Ácido acetóxi- } \\
\text { copálico }\end{array}$ & $\begin{array}{c}\text { Ácido agático } \\
\text { copálico }\end{array}$ \\
\hline 50 & {$[1,671-1,678]$} & {$[1,451-1,462]$} & {$[0,876-0,882]$} & {$[0,705-0,711]$} & {$[0,537-0,541]$} & {$[0,326-0,329]$} & {$[0,183-0,185]$} \\
100 & {$[1,663-1,669]$} & {$[1,442-1,452]$} & {$[0,870-0,877]$} & {$[0,701-0,706]$} & {$[0,533-0,537]$} & {$[0,324-0,327]$} & {$[0,182-0,183]$} \\
150 & {$[1,658-1,664]$} & {$[1,437-1,447]$} & {$[0,867-0,873]$} & {$[0,698-0,703]$} & {$[0,531-0,535]$} & {$[0,323-0,325]$} & {$[0,181-0,183]$} \\
200 & {$[1,655-1,661]$} & {$[1,433-1,443]$} & {$[0,865-0,871]$} & {$[0,696-0,701]$} & {$[0,530-0,534]$} & {$[0,322-0,324]$} & {$[0,181-0,182]$} \\
250 & {$[1.652-1.658]$} & {$[1,430-1,440]$} & {$[0,863-0,869]$} & {$[0,695-0,700]$} & {$[0,529-0,533]$} & {$[0,321-0,324]$} & {$[0,181-0,182]$} \\
300 & {$[1,650-1,656]$} & {$[1,428-1,438]$} & {$[0,861-0,868]$} & {$[0,693-0,699]$} & {$[0,528-0,532]$} & {$[0,321-0,23]$} & {$[0,180-0,181]$} \\
350 & {$[1,648-1,654]$} & {$[1,426-1,436]$} & {$[0,860-0,866]$} & {$[0,692-0,698]$} & {$[0,527-0,531]$} & {$[0,320-0,323]$} & {$[0,180-0,181]$} \\
400 & {$[1,646-1,652]$} & {$[1,424-1,434]$} & {$[0,859-0,865]$} & {$[0,691-0,697]$} & {$[0,526-0,530]$} & {$[0,320-0,322]$} & {$[0,180-0,181]$} \\
450 & {$[1,645-1,651]$} & {$[1,422-1,432]$} & {$[0,858-0,864]$} & {$[0,691-0,696]$} & {$[0,526-0,530]$} & {$[0,320-0,322]$} & {$[0,179-0,181]$} \\
500 & {$[1,643-1,650]$} & {$[1,421-1,431]$} & {$[0,857-0,863]$} & {$[0,690-0,695]$} & {$[0,525-0,529]$} & {$[0,319-0,322]$} & {$[0,179-0,181]$} \\
550 & {$[1,642-1,649]$} & {$[1,420-1,430]$} & {$[0,856-0,863]$} & {$[0,689-0,695]$} & {$[0,525-0,529]$} & {$[0,319-0,321]$} & {$[0,179-0,180]$} \\
600 & {$[1,641-1,648]$} & {$[1,418-1,429]$} & {$[0,856-0,862]$} & {$[0,689-0,694]$} & {$[0,524-0,528]$} & {$[0,319-0,321]$} & {$[0,179-0,180]$} \\
650 & {$[1,640-1,647]$} & {$[1,417-1,428]$} & {$[0,855-0,861]$} & {$[0,688-0,694]$} & {$[0,524-0,528]$} & {$[0,318-0,321]$} & {$[0,179-0,180]$} \\
700 & {$[1,639-1,646]$} & {$[1,416-1,427]$} & {$[0,854-0,861]$} & {$[0,688-0,693]$} & {$[0,523-0,528]$} & {$[0,318-0,320]$} & {$[0,179-0,180]$} \\
750 & {$[1,638-1,645]$} & {$[1,415-1,426]$} & {$[0,854-0,860]$} & {$[0,687-0,693]$} & {$[0,523-0,527]$} & {$[0,318-0,320]$} & {$[0,179-0,180]$} \\
\hline
\end{tabular}

cada biomarcador de acordo com a concentração do óxido de linalol, de modo que se o $\mathrm{Rf}_{\mathrm{R}}$ da substância de um óleo de copaíba qualquer estiver entre os limites especificados na tabela, é possível sugerir a identidade do respectivo biomarcador.

Os valores de $\mathrm{Rf}_{\mathrm{R}}$ apresentados na Tabela 1 foram desenvolvidos a partir das curvas analíticas dos biomarcadores terpênicos em função da concentração do óxido de linalol. No desenvolvimento das curvas analíticas, observou-se um leve aumento nos valores de Rf's obtidos para o óxido de linalol com o aumento da sua concentração. Foi observado que quanto maior a sua concentração, maior foi sua interação com a fase estacionária, contribuindo para o espalhamento da banda, e consequentemente para o aumento da distância central da mancha. Como o $\mathrm{Rf}_{\mathrm{R}}$ consiste na razão entre o $\mathrm{Rf}$ do biomarcador terpênico pelo $\mathrm{Rf}$ do óxido de linalol $\left[\mathrm{Rf}_{\mathrm{R}}=\left(\mathrm{Rf}_{\text {biomarcador }} / \mathrm{Rf}_{\mathrm{O} \text {. Linalol }}\right)\right]$, à medida que a concentração do óxido de linalol aumenta, $o \mathrm{Rf}_{\mathrm{R}}$ do biomarcador terpênico irá diminuir.

\section{Aplicação do método}

A metodologia desenvolvida para caracterização dos biomarcadores terpênicos por meio do fator de retenção relativo $\left(\mathrm{Rf}_{\mathrm{R}}\right)$ foi aplicada a três espécimes de óleos de Copaifera multijuga. Os óleos de copaíba $\left(1 \mathrm{mg} \mathrm{mL}^{-1}\right)$ e o óxido de linalol $\left(100 \mu \mathrm{g} \mathrm{mL}^{-1}\right)$ foram aplicados $(10 \mu \mathrm{L})$ nas placas de HPTLC sob as mesmas condições cromatográficas. $O \beta$-cariofileno $\left[\operatorname{Rf}_{R}=(0,78 / 0,47=1,659)\right]$, o óxido de cariofileno $\left[\operatorname{Rf}_{\mathrm{R}}=(0,68 / 0,47=1,447)\right]$, o ácido copálico $\left[\mathrm{Rf}_{\mathrm{R}}=(0,41 / 0,47=0,872)\right]$ e o acetoxi-copálico $\left[\operatorname{Rf}_{\mathrm{R}}=(0,25 / 0,47=0,532)\right]$ foram caracterizados satisfatoriamente pelo fator de retenção relativo (Figura 3). Os resultados obtidos para os valores de $\mathrm{Rf}_{\mathrm{R}}$ de cada biomarcador nas oleoresinas foram comparados com os limites de predição a 95\% de confiança, disponíveis na tabela 1, a $100 \mu \mathrm{g} \mathrm{mL}^{-1}$ do óxido de linalol. A principal característica dos óleos de $C$. multijuga é a presença majoritária do $\beta$-cariofileno $(\mathrm{Rf}=0,78)$ entre os sesquiterpeno, e do ácido copálico $(\mathrm{Rf}=0,41)$, entre os diterpenos. Além do $\beta$-cariofileno, seu óxido $(\mathrm{Rf}=0,68)$ também é relatado em concentrações variáveis nos óleos dessa espécie. ${ }^{17}$

O ácido copálico é o principal diterpeno detectado nos óleos de
C. multijuga. Entretanto, seus derivados, os ácidos acetóxi-copálico $(\mathrm{Rf}=0,25)$ e hidróxi-copálico, são frequentemente relatados para os óleos dessa espécie. ${ }^{17}$ Os principais biomarcadores terpênicos dos óleos de $C$. multijuga foram caracterizados com êxito, mostrando a grande aplicação do desenvolvimento do $\mathrm{Rf}_{\mathrm{R}}$ em óleos de copaíba por HPTLC.

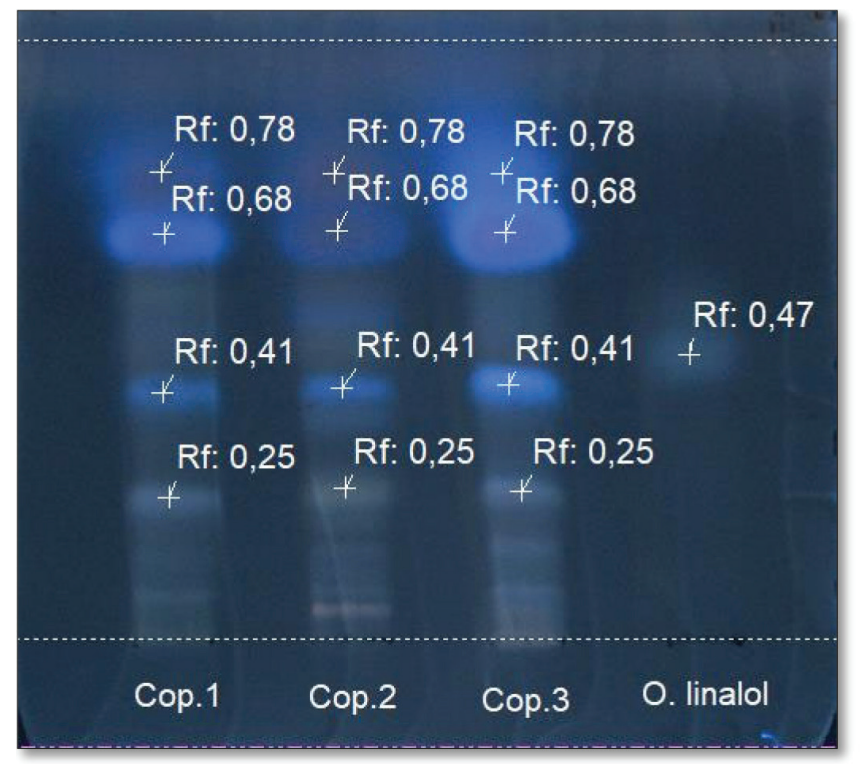

Figura 3. Caracterização química do $\beta$-cariofileno $(R f=0,78)$, óxido de cariofileno $(R f=0,68)$, ácido copálico $(R f=0,41)$ e acetoxi-copálico $(R f=0,25)$ nos óleos de C. multijuga, através do Fator de retenção relativo $\left(R f_{R}\right)$ ao óxido de linalol $\left(100 \mu \mathrm{g} \mathrm{mL}^{-1}\right)$

A metodologia desenvolvida mostrou-se uma excelente alternativa para a caracterização dos biomarcadores dos óleos de $C$. multijuga, principalmente dos ácidos diterpênicos, que são obtidos por isolamento em bancada e usualmente não estão disponíveis comercialmente. Além disso, a utilização do $\mathrm{Rf}_{\mathrm{R}}$ dispensa a derivatização 
das oleoresinas, que é uma etapa obrigatória nas análises por cromatografia à gás e posterga a utilização de técnicas dispendiosas como a HPLC, possibilitando a rápida caracterização dos biomarcadores. Não obstante, $\mathrm{o} \mathrm{Rf}_{\mathrm{R}}$ pode ser aplicado às demais oleoresinas do gênero Copaifera para caracterização de seus biomarcadores terpênicos.

\section{CONCLUSÃO}

O desenvolvimento da metodologia empregando o fator de retenção relativo $\left(\mathrm{Rf}_{\mathrm{R}}\right)$ por HPTLC é descrito pela primeira vez para caracterização de biomarcadores em óleos de copaíba. A metodologia empregando o $\left(\mathrm{Rf}_{\mathrm{R}}\right)$ ao óxido de linalol mostrou-se uma excelente estratégia, de aplicação prática e útil no cotidiano do químico de produtos naturais, por se tratar de um padrão comercial de fácil acesso e baixo custo. A metodologia proporciona identificações rápidas e confiáveis $(\mathrm{CV}<2 \%)$, sem a necessidade do isolamento, possibilitando a caracterização dos constituintes químicos e diferenciação entre os diversos óleos de copaíba.

\section{MATERIAL SUPLEMENTAR}

As curvas analíticas obtidas no desenvolvimento do fator de retenção relativo $\left(\mathrm{Rf}_{\mathrm{R}}\right)$, os parâmetros das curvas e os gráficos da análise de resíduos para ajustes destas curvas estão disponíveis em http://quimicanova.sbq.org.br, em formato pdf, com acesso livre.

\section{AGRADECIMENTOS}

Os autores agradecem ao CNPq, à CAPES, à FAPEAM e à FAPERJ.

\section{REFERÊNCIAS}

1. Veiga Jr., V. F.; Pinto, A. C.; Quim. Nova 2002, 25, 273.

2. Madera, P.; Paschová, Z.; Ansorgová, A.; Vrskovy, B.; Lvoncík, S.; Habrová, H.; Acta Univ. Agric. Silvic. Mendelianae Brun. 2017, 65, 73.

3. Strappaghetti, G.; Corsano, S.; Craveiro, A.; Proietti, G.; Phytochem. 1982, 21, 2114.

4. Siani, A. C.; Nakamura, M. J.; Tappin, M. R. R.; Monteiro, S. S.; Guimarães, A. C.; Ramos, M. F. S.; Phytochem. Anal. 2012, 23, 421.

5. Rudiger, A. L.; Veiga Jr, V. F.; Chem. Biodivers. 2013, 10, 1142.

6. Mercier, B.; Prost, M.; Int. J. Occup. Med. Environ. Health 2009, 22, 331.

7. Keeling, C. I.; Bohlmann, J.; Phytochem. 2006, 67, 2415.

8. Morais, M. M.; Alves, W. S.; Pereira, E. C.; Araújo, A. Z.; Silva, M. A. S.; Vieira, J. S. C.; Resumo do VII CONNEPI, Tocantins, Brasil, 2012.

9. Serra, J. L.; Rodrigues, A. M. C.; De Freitas, R. A.; Meirelles, A. J. A.; Darnet, S. H.; Da Silva, L. H. M.; Food Res. Int. 2019, 116, 12.

10. De Vasconcelos, A. F. F.; Godinho, O. E. S.; Quim. Nova 2002, 25, 1057

11. Zoghbi, M. G. B.; Lameira, O. A.; Oliveira, E. C. P.; J. Essent. Oil Res. 2007, 19, 504; Zoghbi, M. G. B.; Da-Silva, R. C. V. M.; Trigo, J. R.; J. Essent. Oil Res. 2009, 21, 403; Sousa, J. P. B.; Brancalion, A. P. S.; Souza, A. B; Turatti, A. C. C.; Ambrósio, S. R.; Furtado, N. A. J. C.; Lopes, N. P.; Bastos, J. K.; J. Pharm. Biomed. Anal. 2011, 54, 653; Xavier-Junior, F. H.; Maciuk, A.; Morais, A. R. V.; Alencar, E. N.; J.; Chromatogr. Sci. 2017, 55, 969.
12. Patitucci, M. L.; Veiga Jr, V. F.; Pinto, A. C.; Zoghbi, M. G. B.; Silva, J. R. A.; Quim. Nova 1995, 18, 262; Veiga Jr., V. F.; Patitucci, M. L.; Pinto, A. C.; Quim. Nova 1997, 20, 612; Cascon, V.; Gilbert, B.; Phytochemistry 2000, 55, 773; Tappin, M. R. R.; Pereira, J. F. G.; Lima, L. A.; Siani, A. C; Quim. Nova 2004, 27, 236; Biavatti, M. W.; Dossin, D.; Deschamps, F. C.; Lima, M. P.; Rev. Braz. Farmacogn. 2006, 16, 230; Veiga Jr., V. F.; Rosas, E. C.; Carvalho, M. V.; Henriquez, M. G. M. O.; Pinto, A. C.; J. Ethnopharmacol. 2007, 112, 248.

13. Leandro, L. M.; Vargas, F. S.; Barbosa, P. C. S.; Neves, J. K. O.; Da Silva, J. A.; Veiga Jr, V. F.; Molecules 2012, 17, 3866.

14. Boaroto, J. A.; Fernandes, A. B.; Moreira, L. M.; Silveira-Jr., L.; De Lima, C. J.; Instrum. Sci. Technol. 2018, 46, 265.

15. Souza, A. B.; Moreira, M. R.; Borges, C. H. G.; Simão, M. R.; Bastos, J. K.; Sousa, J. P. B.; Ambrosio, S. R.; Veneziani, R. C. S.; Biomed. Chromatogr. 2013, 27, 280; Da Silva, J. J. M.; Crevelin, E. J.; Carneiro, L. J.; Rogez, H.; Veneziani, R. C. S.; Ambrósio, S. R.; Moraes, L. A. B.; Bastos, J. K.; J. Chromatogr. A 2017, 1515, 81; Carneiro, L. J.; Bianchi, T. C.; Da Silva, J. J. M.; Oliveira, L. C.; Borges, C. H. G.; Lemes, D. C.; Bastos, J. K.; Veneziani, R. C. S.; Ambrósio, S. R.; J. Braz. Chem. Soc. 2018, 29, 729; Çiçek, S. S.; Barbosa, A. L. P; Girreser, U.; J. Pharm. Biomed. Anal. 2018, 160, 126.

16. Bardají, D. K. R.; Da Silva, J. J. M.; Bianchi, T. C.; Eugênio, D. S.; De Oliveira, P. F.; Leandro, L. F.; Rogez, H. L. G.; Venezianni, R. C. S.; Ambrosio, S. R.; Tavares, D. C.; Bastos, J. K.; Martins, C. H. G.; Anaerobe 2016, 40, 18.

17. Barbosa, P. C. S.; Medeiros, R. S.; Sampaio, P. T. B.; Vieira, G.; Wiedemann, L. S. M.; Veiga Jr., V. F.; J. Braz. Chem. Soc. 2012, 23, 1823; Barbosa, P. C. S.; Wiedemann, L. S. M.; Medeiros, R. S.; Sampaio, P. T. B.; Vieira, G.; Veiga Jr., V. F.; Chem. Biodiversity 2013, 10, 1350; De Lima, M. C.; Da Silva, L. S.; Veiga Jr, V.F.; Wiedemann, L. S. M.; Braz. J. Dev. 2020, 06, 608.

18. Kovats, V. E.; Helv. Chim. Acta 1958, 41, 1915; van Den Dool, H; Kratz, P. D.; J Chromatogr. A 1963, 11, 463; Krupcik, J.; Repka, D.; Revesi, T.; Anders, G.; J. Chromatogr. 1987, 407, 65; Babushok, V. I.; Trends Anal. Chem. 2015, 69, 98 .

19. De Barros, I. B.; Ribeiro, C. V.; Veiga Jr., V. F.; Quim. Nova 2015, 38, 1192.

20. Makowicz, E.; Jasicka-Misiak, I.; Teper, D.; Kafarski, P.; Molecules 2018, 23, 1811; Shawky, E.; Newehy, N. M. E.; Beltagy, A. M.; AbdAlhaseeb, M. M.; Omran, G. A; Harraz, F. M.; J. Chromatogr. B 2018, 1087, 108; Wang, P.; Chen, Y.; Xu, X.; Hellmann, B.; Huang, C.; Bai, Y.; Jin, Z.; Food Anal. Methods 2019, 12, 431; Rouhani, M.; Ind. Crops. Prod. 2019, 132, 226; Frommenwiler, D. A.; Booker, A.; Vila, R.; Heinrich, M.; Reich, E.; Cañigueral, S.; J. Ethnopharmacol. 2019, 243, 112084.

21. De Souza, F. C.; Tese de Doutorado, Universidade Federal do Amazonas, Brasil, 2018.

22. R Development Core Team: $R$ A Language and Environment for Statistical Computing. R Foundation for Statistical Computing, Vienna, 2018.

23. Trindade, R.; Da Silva, J. K.; Setzer, W. N.; Int. J. Mol. Sci. 2018, 19, 1511.

24. Galúcio, C. S.; Benites, C. I.; Rodrigues, R. A. F.; Maciel, M. R. W.; Quim. Nova 2016, 39, 795. 